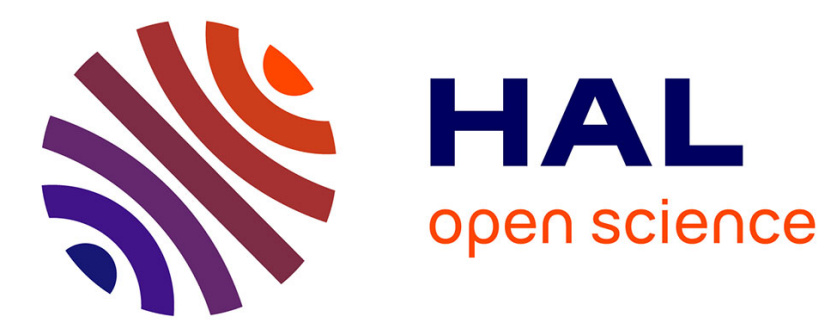

\title{
OPTIMAL CONTROLLABILITY FOR SCALAR CONSERVATION LAWS WITH CONVEX FLUX
}

Adi Adimurthi, Shyam Sundar Ghoshal, Veerappa Gowda

\section{To cite this version:}

Adi Adimurthi, Shyam Sundar Ghoshal, Veerappa Gowda. OPTIMAL CONTROLLABILITY FOR SCALAR CONSERVATION LAWS WITH CONVEX FLUX. 2012. hal-00805317

\author{
HAL Id: hal-00805317 \\ https://hal.science/hal-00805317 \\ Preprint submitted on 28 Mar 2013
}

HAL is a multi-disciplinary open access archive for the deposit and dissemination of scientific research documents, whether they are published or not. The documents may come from teaching and research institutions in France or abroad, or from public or private research centers.
L'archive ouverte pluridisciplinaire HAL, est destinée au dépôt et à la diffusion de documents scientifiques de niveau recherche, publiés ou non, émanant des établissements d'enseignement et de recherche français ou étrangers, des laboratoires publics ou privés. 


\title{
OPTIMAL CONTROLLABILITY FOR SCALAR CONSERVATION LAWS WITH CONVEX FLUX
}

\author{
ADIMURTHI*, SHYAM SUNDAR GHOSHAL ${ }^{\dagger}$, AND G. D. VEERAPPA GOWDA
}

\begin{abstract}
In this paper we derive a method to obtain a solution of an optimal control problem for a scalar conservation laws with convex flux. By using the method of descent, this type of problem was considered by Castro-Palacios-Zuazua in [5] for the Burgers equation. Our approach is simple and based on the explicit formulas of Hopf and Lax-Olenik.
\end{abstract}

Key words: Hamilton-Jacobi equation, scalar conservation laws, characteristics, explicit formulas, optimal controllability.

1. Introduction. Let $f: \mathbb{R} \rightarrow \mathbb{R}$ be $C^{1}$, strictly convex function having super linear growth. That is

$$
\lim _{|u| \rightarrow \infty} \frac{f(u)}{|u|}=\infty
$$

Let $u_{0} \in L^{\infty}(\mathbb{R})$ and consider the scalar conservation law

$$
\begin{aligned}
& u_{t}+f(u)_{x}=0, \quad \text { if } x \in \mathbb{R}, t>0 \\
& u(x, 0) \\
& =u_{0}(x) .
\end{aligned}
$$

In general, (1.2) does not admit classical solutions and hence we look for weak solutions. This problem was well studied and showed that (1.2) admits a unique weak solution satisfying Lax-Olenik-Kruzkov entropy conditions. In the sequel we mean $u$ a solution of (1.2) if it is a weak solution satisfying entropy condition.

In [5] the following optimal control problem associated to (1.2) had been considered. Let $k \in L_{l o c}^{2}(\mathbb{R})$ be a target function and $A \subset L^{\infty}(\mathbb{R})$, a set of admissible controls. Let $u$ be the associated solution of (1.2) with initial data $u_{0}$ and $T>0$. Define the cost functional $\tilde{J}$ on A by

$$
\tilde{J}\left(u_{0}\right)=\int_{-\infty}^{\infty}|u(x, T)-k(x)|^{2} d x .
$$

Then the optimal control problem is to find an $u_{0} \in A$ such that

$$
\tilde{J}\left(u_{0}\right)=\min _{w_{o} \in A} \tilde{J}\left(w_{0}\right) .
$$

Under a suitable conditions on $\mathrm{k}$ and $\mathrm{A}$, they prove that $u_{0}$ exists. In general $u_{0}$ is not unique. The basic problem related to this is "to capture a minimizer". It is a very hard problem because the cost functional $\tilde{J}$ is highly nonlinear, non differentiable and non convex. For the Burger's equation, in [5], [6] they have proposed a numerical scheme called "alternating descent direction" by using the linearization technique developed in [4],[3]. In that work, convergence analysis is completely open.

In this paper, we tackle this problem in a completely different way. In view of the non linearity, we modify the cost function $\tilde{J}$ to $J$ so that the optimal control

\footnotetext{
*TIFR-CAM, Bangalore - 560065, India, (aditi@math.tifrbng.res.in).

†TIFR-CAM, Bangalore - 560065, India, (ssghoshal@math.tifrbng.res.in).

¥TIFR-CAM, Bangalore - 560065, India,(gowda@math.tifrbng.res.in).
} 
problem reduces to the standard convex optimization problem via Lax-Oleinik explicit formula. Then we use the backward algorithm introduced in [1],[2] to obtain an optimal solution. The novelty of this method is that it is constructive and easy to derive a numerical scheme to capture an optimal solution.

Authors would like to thank Enrique Zuazua for providing valuable references.

In the forthcoming paper, these results have been extended to conservation laws with discontinuous flux.

2. Preliminaries. Before stating the main results, let us recall Hopf, LaxOleinik explicit formulas (see [7]).

Let $u_{0} \in L^{\infty}(\mathbb{R})$ and $f$ be as in the introduction. let $f^{*}$ denote its convex dual defined by

$$
f^{*}(p)=\sup _{q}\left\{p q-f^{*}(q)\right\}
$$

then $f^{*}$ is $C^{1}$, superlinear growth and satisfies

$$
\begin{array}{ll}
f(p) & =\sup _{q}\left\{p q-f^{*}(q)\right\} \\
f^{*}\left(f^{\prime}(p)\right) & =p f^{\prime}(p)-f(p), \\
f^{* \prime} & =f^{\prime-1} .
\end{array}
$$

Let $b \in \mathbb{R}$ and define $v_{0}$ by

$$
v_{0}(x)=\int_{b}^{x} u_{0}(y) d y,
$$

and the associated value function $v$ is given by

$$
v(x, t)=\inf _{y}\left\{v_{0}(y)+t f^{*}\left(\frac{x-y}{t}\right)\right\} .
$$

Definition 2.1 (Characteristic): Define the characteristic set ch( $x, t)$, extreme characteristics $y_{ \pm}(x, t)$ by

$$
\begin{aligned}
& \operatorname{ch}(x, t)=\{y: y \text { is a minimizer in }(2.4)\} \\
& y_{-}(x, t)=\inf \{y: y \text { is in ch }(x, t)\} \\
& y_{+}(x, t)=\sup \{y: y \text { is in } \operatorname{ch}(x, t)\}
\end{aligned}
$$

Then we have the following theorem due to Hopf, Lax-Oleinik ( see [7]).

Theorem 2.2. (1). $v$ is a uniformly Lipschitz continuous function with Lipschitz constant depending only on $\left\|u_{0}\right\|_{\infty}, f^{*}$ and satisfies the Hamilton-Jacobi equation

$$
\begin{aligned}
v_{t}+f\left(v_{x}\right) & =0 \quad x \in \mathbb{R}, t>0, \\
v(x, 0) & =v_{0}(x)
\end{aligned}
$$

in the sense of viscosity.

(2). $\operatorname{ch}(x, t) \neq \phi$ and there exists $M>0$ depending only on $\left\|u_{0}\right\|_{\infty}$ and $f^{*}$ such that for all $(x, t) \in \mathbb{R} \times \mathbb{R}_{+}, y \in \operatorname{ch}(x, t)$

$$
\left|\frac{x-y}{t}\right| \leq M
$$


(3). For each time $t, x \mapsto y_{ \pm}(x, t)$ are non decreasing functions and for a.e. $x$,

$$
y_{+}(x, t)=y_{-}(x, t) .
$$

(4). Let $u=\frac{\partial v}{\partial x}$, then $u$ is the solution of (1.2) such that for a.e. $x, t>0$,

$$
f^{\prime}(u(x, t))=\frac{x-y_{+}(x, t)}{t}=\frac{x-y_{-}(x, t)}{t} .
$$

Furthermore

$$
u(x, t)=u_{0}\left(y_{ \pm}(x, t)\right)
$$

if $x$ is a point of differentiability of $y_{ \pm}(x, t)$ and $y_{ \pm}(x, t)$ is a point of differentiability for $v_{0}$.

As an immediate consequence of this theorem, we have the following Lemma on finite speed of propagation.

Lemma 2.3. Let $A_{1}<A_{2}, u_{-}, u_{+} \in \mathbb{R}, \bar{u}_{0} \in L^{\infty}\left(A_{1}, A_{2}\right)$. Define $u_{0}$ by

$$
u_{0}(x)= \begin{cases}u_{-} & \text {if } x<A_{1}, \\ \bar{u}_{0}(x) & \text { if } A_{1}<x<A_{2}, \\ u_{+} & \text {if } x>A_{2} .\end{cases}
$$

Then for $t>0$, the solution $u$ satisfies

$$
u(x, t)= \begin{cases}u_{-} & \text {if } x<A_{1}-M t, \\ u_{+} & \text {if } \quad x>A_{2}+M t .\end{cases}
$$

Proof. Let $t>0$, then from (2.10), if $x<A_{1}-M t$, then

$$
-M \leq \frac{x-y_{ \pm}(x, t)}{t}<\frac{A_{1}-M t-y_{ \pm}(x, t)}{t} .
$$

Hence $y_{ \pm}(x, t)<A_{1}$. Since $v_{0}$ is differentiable in $\left(-\infty, A_{1}\right)$ and hence from (2.12), at the differentiable point $x$ of $y_{ \pm}(x, t)$, we have

$$
u(x, t)=u_{0}\left(y_{ \pm}(x, t)\right)=u_{-} .
$$

Similarly if $x>A_{2}+M t$, then for a.e. $x, u(x, t)=u_{+}$. This proves (2.14) and hence the Lemma.

3. Main results. Let

$$
f\left(\theta_{f}\right)=\min _{\theta \in \mathbb{R}} f(\theta) .
$$

Define

1. Admissible sets $A$ and $B$ :

$$
\begin{aligned}
& A=\left\{u_{0} \in L^{\infty}(\mathbb{R}): u_{0}(x)=\theta_{f} \text { outside a compact set }\right\} \\
& B=\left\{\rho \in L_{\text {loc }}^{\infty}(\mathbb{R}): \begin{array}{ll}
\text { (i) } \rho \text { is a non decreasing function } \\
& \text { (ii) } \rho(x)=x \text { outside a compact set }\} .
\end{array}\right.
\end{aligned}
$$

For each $N>0$, define

$$
B_{N}=\{\rho \in B: \rho(x)=x \text { for }|x|>N\} .
$$


2. Target function $k$ : Let $I=\left(C_{1}, C_{2}\right)$ and $k$ be a measurable function such that

$$
\begin{aligned}
k(x) & =\theta_{f} \quad \text { if } \quad x \notin\left(C_{1}, C_{2}\right), \\
f^{\prime}(k(x)) & \in L^{2}(I) .
\end{aligned}
$$

3. Cost function $J$ : Let $k$ be a target function and $u_{0} \in A$. Let $u(x, t)$ be the corresponding solution of (1.2) with initial data $u_{0}$. Let $T>0$ and define the modified cost function

$$
J\left(u_{0}\right)=\int_{\mathbb{R}}\left|f^{\prime}(u(x, T))-f^{\prime}(k(x))\right|^{2} d x .
$$

Then we have the following proposition.

Proposition 3.1. $J$ is well defined.

Proof. Choose $A_{1}<C_{1}<C_{2}<A_{2}$ such that $u_{0}(x)=\theta_{f}$ for $x \notin\left(A_{1}, A_{2}\right)$. Then from Lemma (2.3), there exists an $M>0$ depending only on $\left\|u_{0}\right\|_{\infty}$ and $f^{*}$ such that

$$
u(x, T)=\theta_{f} \quad \text { for } \quad x \notin\left[A_{1}-M T, A_{2}+M T\right]
$$

and hence $f^{\prime}(u(x, T))=f^{\prime}\left(\theta_{f}\right)=0$. Hypothesis on $k$ implies for $x \notin\left[C_{1}, C_{2}\right]$

$$
f^{\prime}(k(x))=f^{\prime}\left(\theta_{f}\right)=0 .
$$

Hence

$$
J\left(u_{0}\right)=\int_{A_{1}-M T}^{A_{2}+M T}\left|f^{\prime}(u(x, T))-f^{\prime}(k(x))\right|^{2} d x .
$$

This proves the proposition.

Optimal control problem : Given $A, k$ as above, find a $\tilde{u}_{0} \in A$ such that

$$
J\left(\tilde{u}_{0}\right)=\min _{u_{0} \in A} J\left(u_{0}\right)
$$

and if the minimizer exist, then device a scheme to capture it.

Then we have the following main result.

Theorem 3.2. There exists a minimizer for (3.10) which can be captured by using the standard convex optimization problem in a Hilbert space and backword algorithm.

This Theorems has been generalized to $L^{p}$ cost functional (see section 4).

In order to prove this Theorem, first we reduce the problem to a standard projection Theorem in a Hilbert space and then use the backward algorithm.

Reduction to a projection on a convex set : Let $y_{+}(x, t)$ be the right extreme characteristic corresponding to the solution $u$. Let

$$
\rho(x)=y_{+}(x, T), \quad x \in \mathbb{R},
$$

then $\rho$ is a non decreasing function and from (2.11) for a.e. $x$,

$$
f^{\prime}(u(x, T))=\frac{x-\rho(x)}{T} .
$$


Since $u(x, T)=\theta_{f}$ for $x \notin\left[A_{1}-M T, A_{2}+M T\right]$, hence $\rho(x)=x$ for $x \notin\left[A_{1}-\right.$ $\left.M T, A_{2}+M T\right]$ and

$$
\begin{aligned}
J\left(u_{0}\right) & =\int_{-\infty}^{\infty}\left|\frac{x-\rho(x)}{T}-f^{\prime}(k(x))\right|^{2} d x \\
& =\int_{A_{1}-M T}^{A_{2}+M T}\left|\frac{x-\rho(x)}{T}-f^{\prime}(k(x))\right|^{2} d x .
\end{aligned}
$$

Now define $\bar{J}$ on $B$ by

$$
\bar{J}(\rho)=\int_{-\infty}^{\infty}\left|\frac{x-\rho(x)}{T}-f^{\prime}(k(x))\right|^{2} d x .
$$

Then from (3.11) to (3.13) we have

$$
\inf _{\rho \in B} \bar{J}(\rho) \leq \inf _{u_{0} \in A} J\left(u_{0}\right) .
$$

Lemma 3.3. Let

$$
\tilde{B}=\left\{\rho \in B: \rho(x)=x \text { if } x \notin\left[\min \left(C_{1}, \rho\left(C_{1}\right)\right), \max \left(C_{2}, \rho\left(C_{2}\right)\right)\right]\right\}
$$

Then

$$
\inf _{\rho \in B} \bar{J}(\rho)=\inf _{\tilde{\rho} \in \tilde{B}} \bar{J}(\tilde{\rho})
$$

Proof. Since $\tilde{B} \subset B$, hence

$$
\inf _{\tilde{\rho} \in \tilde{B}} \bar{J}(\tilde{\rho}) \geq \inf _{\rho \in B} \bar{J}(\rho) .
$$

Let $\rho \in B$ and define $\tilde{\rho}$ by

$$
\tilde{\rho}(x)=\left\{\begin{array}{lll}
x & \text { if } & x \notin\left[\min \left(C_{1}, \rho\left(C_{1}\right)\right), \max \left(C_{2}, \rho\left(C_{2}\right)\right)\right] \\
\rho\left(C_{1}\right) & \text { if } & \min \left(C_{1}, \rho\left(C_{1}\right)\right) \leq x<C_{1} \\
\rho(x) & \text { if } & x \in\left[C_{1}, C_{2}\right] \\
\rho\left(C_{2}\right) & \text { if } & C_{2}<x<\max \left(C_{2}, \rho\left(C_{2}\right)\right) .
\end{array}\right.
$$

Then

$$
\begin{aligned}
\bar{J}(\tilde{\rho}) & =\int_{\min \left(C_{1}, \rho\left(C_{1}\right)\right)}^{\max \left(C_{2}, \rho\left(C_{2}\right)\right)}\left|\frac{x-\tilde{\rho}(x)}{T}-f^{\prime}(k(x))\right|^{2} d x \\
& =\int_{C_{1}}^{C_{2}}\left|\frac{x-\rho(x)}{T}-f^{\prime}(k(x))\right|^{2} d x+\int_{\min \left(C_{1}, \rho\left(C_{1}\right)\right)}^{C_{1}}\left|\frac{x-\rho\left(C_{1}\right)}{T}\right|^{2} d x \\
& +\int_{C_{2}}^{\max \left(C_{2}, \rho\left(C_{2}\right)\right)}\left|\frac{x-\rho\left(C_{2}\right)}{T}\right|^{2} d x .
\end{aligned}
$$


Since $\rho(x) \leq \rho\left(C_{1}\right)$ for $x \in\left(\min \left(C_{1}, \rho\left(C_{1}\right)\right), C_{1}\right)$ and $\rho(x) \geq \rho\left(C_{2}\right)$ for $x \in\left(C_{2}\right.$, $\left.\max \left(C_{2}, \rho\left(C_{2}\right)\right)\right)$, hence

$$
\begin{aligned}
\bar{J}(\tilde{\rho}) & \leq \int_{C_{1}}^{C_{2}}\left|\frac{x-\rho(x)}{T}-f^{\prime}(k(x))\right|^{2} d x \\
& +\int^{C_{1}}\left|\frac{x-\rho(x)}{T}\right|^{2} d x+\int_{C_{2}}^{\max \left(C_{2}, \rho\left(C_{2}\right)\right)}\left|\frac{x-\rho(x)}{T}\right|^{2} d x \\
& \leq \int_{-\infty}^{\infty}\left|\frac{x-\rho(x)}{T}-f^{\prime}(k(x))\right|^{2} d x \\
& =\bar{J}(\rho) .
\end{aligned}
$$

Hence

$$
\inf _{\tilde{\rho} \in \tilde{B}} \bar{J}(\tilde{\rho}) \leq \inf _{\rho \in B} J(\rho) .
$$

This proves the Lemma.

Lemma 3.4. Let

$$
\begin{aligned}
& \tilde{C}_{1}=C_{1}-\left(3 T^{2}\left(\left\|f^{\prime}(k)\right\|_{2}^{2}+1\right)\right)^{1 / 3} \\
& \tilde{C}_{2}=C_{2}+\left(3 T^{2}\left(\left\|f^{\prime}(k)\right\|_{2}^{2}+1\right)\right)^{1 / 3} \\
& \tilde{B}_{0}=\left\{\rho \in \tilde{B}: \rho(x)=x \text { for } x \notin\left[\tilde{C}_{1}, \tilde{C}_{2}\right]\right\},
\end{aligned}
$$

then there exists an unique $\tilde{\rho}_{0} \in \tilde{B}_{0}$ such that

$$
\bar{J}\left(\tilde{\rho}_{0}\right)=\min _{\tilde{\rho} \in \tilde{B}_{0}} \bar{J}(\tilde{\rho})=\min _{\rho \in B} \bar{J}(\rho) .
$$

Proof. Let $\tilde{\rho}_{k} \in \tilde{B}$ be such that

$$
\lim _{k \rightarrow \infty} \bar{J}\left(\tilde{\rho}_{k}\right)=\inf _{\tilde{h} \in \tilde{B}} \bar{J}(\tilde{\rho})
$$

Let $\eta(x)=x$ for all $x \in \mathbb{R}$, then $\eta \in \tilde{B}$ and

$$
\begin{aligned}
\left\|f^{\prime}(k)\right\|_{2}^{2} & =\bar{J}(\eta) \geq \lim _{k \rightarrow \infty} \bar{J}\left(\tilde{\rho}_{k}\right) \\
& \geq \lim _{k \rightarrow \infty}\left\{\int_{\min \left(C_{1}, \rho_{k}\left(C_{1}\right)\right)}^{C_{1}}\left|\frac{x-\rho_{k}(x)}{T}\right|^{2} d x+\int_{C_{2}}^{\max \left(C_{2}, \rho_{k}\left(C_{2}\right)\right)}\left|\frac{x-\tilde{\rho}_{k}(x)}{T}\right|^{2} d x\right\} .
\end{aligned}
$$

Since

$$
\tilde{\rho}_{k}(x)=\left\{\begin{array}{l}
\tilde{\rho}_{k}\left(C_{1}\right) \text { if } x \in\left(\min \left(C_{1}, \tilde{\rho}_{k}\left(C_{1}\right)\right), C_{1}\right), \\
\tilde{\rho}_{k}\left(C_{2}\right) \text { if } x \in\left(C_{2}, \max \left(C_{2}, \rho_{k}\left(C_{2}\right)\right)\right)
\end{array}\right.
$$


and hence there exists $k_{0}>0$ such that for all $k \geq k_{0}$,

$$
\begin{aligned}
3 T^{2}\left(\left\|f^{\prime}(k)\right\|_{2}^{2}+1\right) & \geq 3 T^{2} \int_{\min \left(C_{1}, \tilde{\rho}_{k}\left(C_{1}\right)\right)}^{C_{1}}\left(\frac{x-\tilde{\rho}_{k}\left(C_{1}\right)}{T}\right)^{2} d x \\
& +3 T^{2} \int_{C_{2}}^{\max \left(C_{2}, \tilde{\rho}_{k}\left(C_{2}\right)\right)}\left(\frac{x-\tilde{\rho}_{k}\left(C_{2}\right)}{T}\right)^{2} \\
& =\left(C_{1}-\min \left(C_{1}, \tilde{\rho}_{k}\left(C_{1}\right)\right)\right)^{3}+\left(\max \left(C_{2}, \tilde{\rho}_{k}\left(C_{2}\right)\right)-C_{2}\right)^{3} .
\end{aligned}
$$

Hence

$$
\begin{aligned}
\min \left(C_{1}, \tilde{\rho}_{k}\left(C_{1}\right)\right) & \geq \tilde{C}_{1} \\
\max \left(C_{2}, \tilde{\rho}_{k}\left(C_{2}\right)\right) & \leq \tilde{C}_{2} .
\end{aligned}
$$

This implies that $\tilde{\rho}_{k}\left(C_{1}\right), \tilde{\rho}_{k}\left(C_{2}\right) \in\left[\tilde{C}_{1}, \tilde{C}_{2}\right]$ and hence

$$
\inf _{\tilde{\rho} \in \tilde{B}} \bar{J}(\tilde{\rho})=\inf _{\tilde{\rho} \in \tilde{B}_{0}} \bar{J}(\tilde{\rho}) .
$$

Let

$$
H=L^{2}\left(\tilde{C}_{1}, \tilde{C}_{2}\right), K=\left\{\rho \in \tilde{B}: \tilde{C}_{1} \leq \min \left(C_{1}, \rho\left(C_{1}\right)\right) \leq \max \left(C_{2}, \rho\left(C_{2}\right)\right) \leq \tilde{C}_{2}\right\} .
$$

Then $K$ is a closed convex set in $H$ and hence from the projection Theorem in a Hilbert space, there exists an unique $\tilde{\rho}_{0} \in K$ such that

$$
\bar{J}\left(\tilde{\rho}_{0}\right)=\min _{\tilde{\rho} \in K} \bar{J}(\tilde{\rho}) .
$$

This proves the Lemma.

In order to prove the main results, we need the following backward algorithm. In [1] we have generalized this to several other cases where it play an imprtant role in proving the exact controllability. This construction is not unique and one can have infinitely many datas which gives the same solution $u(\cdot, T)$ (see [1]).

Lemma 3.5.(Backward algorithm): Let $\tilde{C}_{1}<\tilde{C}_{2}, \tilde{\rho}_{0}$ be as in Lemma 3.4. Then there exists an $\tilde{u}_{0} \in L^{\infty}(\mathbb{R}), \tilde{u} \in L^{\infty}\left(\mathbb{R} \times \mathbb{R}_{+}\right)$such that $\tilde{u}$ is the solution of (1.1) with initial data $\tilde{u}_{0}$. Moreover $\tilde{u}$ satisfies

$$
f^{\prime}(\tilde{u}(x, T))=\frac{x-\tilde{\rho}_{0}}{T}
$$

and the construction of $\tilde{u}_{0}, \tilde{u}$ are algorithmimic and easy to compute.

Proof. Without loss of generality we can assume that $\tilde{\rho}_{0}:\left[\tilde{C}_{1}, \tilde{C}_{2}\right] \rightarrow\left[\tilde{C}_{1}, \tilde{C}_{2}\right]$ is a non decreasing left continuous function with $\tilde{\rho}_{0}\left(\tilde{C}_{i}\right)=\tilde{C}_{i}$ for $i=1,2$.

Step 1 : Discretization of $\tilde{\rho}_{0}$ : Let $n \geq 1$ and $\tilde{C}_{1}=y_{0}<y_{1}<\cdots<y_{n}=\tilde{C}_{2}$ be such that $\left|y_{i}-y_{i+1}\right| \leq 1 / n$. From the left continuity, define $\tilde{C}_{1}=x_{0} \leq x_{1} \leq \cdots \leq x_{n}=\tilde{C}_{2}$ by $\tilde{\rho}_{0}^{-1}\left[y_{0}, y_{i}\right]=\left[x_{0}, x_{i}\right]$ and

$$
\rho_{n}(x)=\left\{\begin{array}{lll}
x & \text { if } & x \notin\left[\tilde{C}_{1}, \tilde{C}_{2}\right] \\
y_{0} \chi_{\left[y_{0}, y_{1}\right]}(x)+\sum_{i=1}^{n-1} y_{i} \chi_{\left(y_{i}, y_{i+1}\right]}(x) & \text { if } & x \in\left[\tilde{C}_{1}, \tilde{C}_{2}\right] .
\end{array}\right.
$$


Then for $x \in \mathbb{R}$,

$$
\left|\rho_{n}(x)-\tilde{\rho}_{0}(x)\right| \leq 1 / n \text {. }
$$

Step 2 : Define $a_{i, n}, b_{i, n}, s_{i, n}, a_{i, n}(t), b_{i, n}(t), s_{i, n}(t)$ (See Figure 3.1) by

$$
\begin{aligned}
f^{\prime}\left(a_{i, n}\right) & =\frac{y_{i}-x_{i}}{T} \\
f^{\prime}\left(b_{i, n}\right) & =\frac{y_{i}-x_{i+1}}{T} \\
f^{\prime}\left(s_{i, n}\right) & =\frac{f\left(b_{i, n}\right)-f\left(a_{i, n}\right)}{b_{i, n}-s_{i, n}} \\
a_{i, n}(t) & =y_{i, n}+f^{\prime}\left(a_{i, n}\right)(t-T) \\
b_{i, n}(t) & =y_{i, n}+f^{\prime}\left(b_{i, n}\right)(t-T) \\
s_{i, n}(t) & =y_{i, n}+f^{\prime}\left(s_{i, n}\right)(t-T) .
\end{aligned}
$$

Now $f^{\prime}\left(a_{i, n}\right)=\frac{y_{i}-x_{i}}{T} \geq \frac{y_{i}-x_{i+1}}{T}=f^{\prime}\left(b_{i, n}\right)$ and $f^{\prime}\left(b_{i, n}\right)=\frac{y_{i}-x_{i+1}}{T} \leq \frac{y_{i+1}-x_{i+1}}{T}=$ $f^{\prime}\left(a_{i+1, n}\right)$. Hence $b_{i, n} \leq \min \left\{a_{i, n}, a_{i+1, n}\right\}$ and from convexity $a_{i, n} \geq s_{i, n} \geq b_{i, n}$. Therefore for all $0 \leq t \leq T, a_{i, n}(t) \leq s_{i, n}(t) \leq b_{i, n}(t)$. Let $s_{i, n}(0)=\alpha_{i}$ and for $(x, t) \in \mathbb{R} \times[0, T]$, define

$$
f^{\prime}\left(u_{n}(x, t)\right)=\left\{\begin{array}{lll}
f^{\prime}\left(a_{i, n}\right) & \text { if } & a_{i, n}(t)<x<s_{i, n}(t) \\
f^{\prime}\left(b_{i, n}\right) & \text { if } & s_{i, n}(t)<x<b_{i, n}(t) \\
\frac{x_{i+1}-y}{t} & \text { if } & b_{i, n}(t)<x<a_{i+1, n}(t) .
\end{array}\right.
$$

Since $a_{0, n}=a_{n, n}=\theta_{f}$, we have

$$
f^{\prime}\left(u_{n}(x, t)\right)=0 \quad \text { if } \quad x \notin\left[\tilde{C}_{1}, \tilde{C}_{2}\right], 0 \leq t \leq T .
$$

Then clearly $u_{n}$ satisfies

$$
\begin{aligned}
u_{n_{t}}+f\left(u_{n}\right)_{x} & =0 \quad \mathbb{R} \times(0, T) \\
f^{\prime}\left(u_{n}(x, t)\right) & =\left\{\begin{array}{llc}
\frac{x-\rho_{n}(x)}{t} & \text { if } \tilde{C}_{1}<x<\tilde{C}_{2} \\
0 & \text { if } & x \notin\left[\tilde{C}_{1}, \tilde{C}_{2}\right] .
\end{array}\right.
\end{aligned}
$$

Also

$$
u_{n}(x, 0)=\left\{\begin{array}{lll}
a_{i, n} & \text { if } & a_{i, n}<x<s_{i, n}(0) \\
b_{i, n} & \text { if } & s_{i, n}(0)<x<a_{i+1, n} \\
\theta_{f} & \text { if } & x \notin\left[\tilde{C}_{1}, \tilde{C}_{2}\right]
\end{array}\right.
$$

Hence

$$
\begin{aligned}
\left|f^{\prime}\left(u_{n}(x, 0)\right)\right| & \leq \operatorname{Max}\left\{\left|f^{\prime}\left(a_{i, n}\right)\right|,\left|f^{\prime}\left(b_{i, n}\right)\right|\right\} \\
& \leq \frac{\left|\tilde{C}_{2}-\tilde{C}_{1}\right|}{T}
\end{aligned}
$$

and

$$
\begin{aligned}
T V\left(f^{\prime}\left(u_{n}(x, 0)\right)\right) & \leq \sum\left|f^{\prime}\left(a_{i, n}\right)-f^{\prime}\left(b_{i, n}\right)\right|+\sum\left|f^{\prime}\left(b_{i, n}\right)-f^{\prime}\left(a_{i+1, n}\right)\right| \\
& =\sum\left|\frac{x_{i}-x_{i+1}}{T}\right|+\sum\left|\frac{y_{i}-y_{i+1}}{T}\right| \\
& \leq \frac{2\left(\tilde{C}_{2}-\tilde{C}_{1}\right)}{T}
\end{aligned}
$$




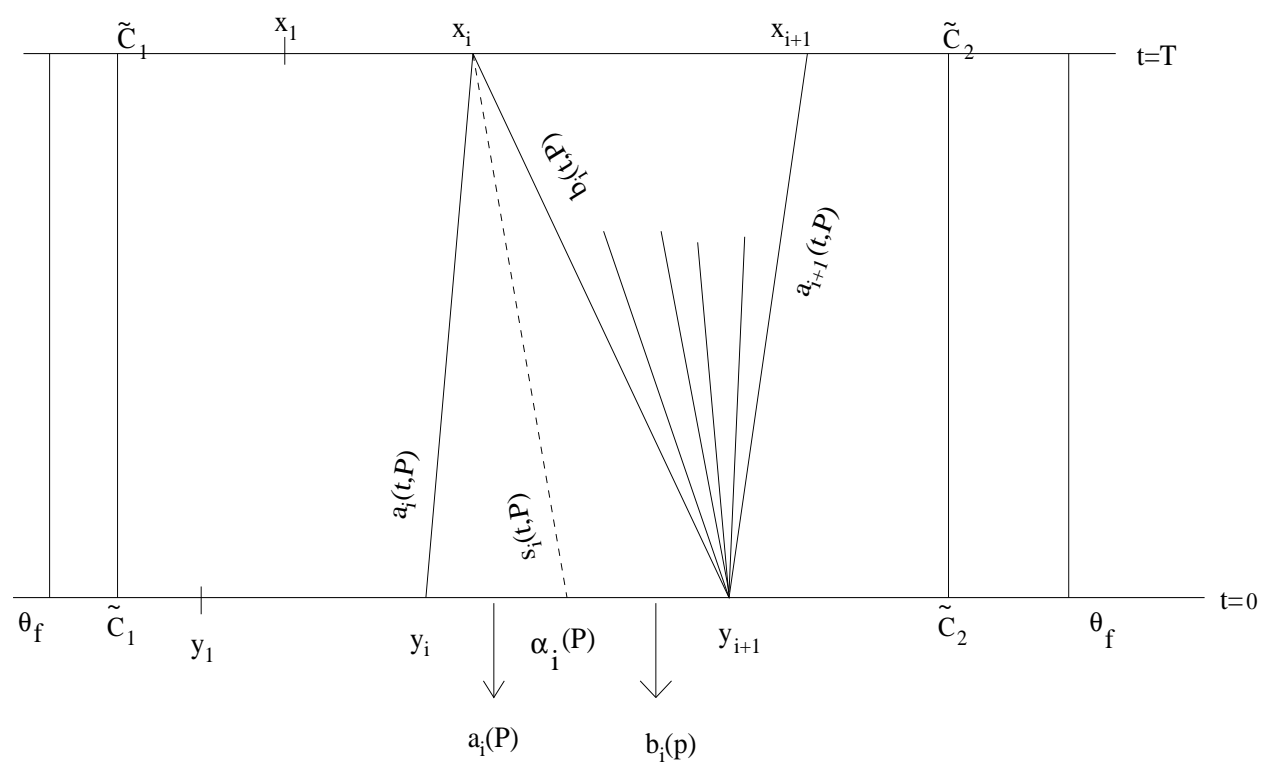

FIG. 3.1.

Hence from Helley's theorem, there exist a subsequence still denoted $\left\{f^{\prime}\left(u_{n}(\cdot, 0)\right)\right\}$ converges to a function $z$ pointwise. Let $\tilde{u}_{0}(x)=\left(f^{\prime}\right)^{-1}(z(x))$, hence $u_{n}(x, 0) \rightarrow$ $\tilde{u}_{0}(x)$. From super linear growth, $\left\{u_{n}(\cdot, 0)\right\}$ are uniformly bounded, hence $\tilde{u}_{0} \in L^{\infty}(\mathbb{R})$. Further more by construction, $u_{n}$ satisfies

$$
f^{\prime}\left(u_{n}(x, T)\right)=\frac{x-\rho_{n}(x)}{T} .
$$

Let $\tilde{u}$ be the solution of (1.1) with $\tilde{u}_{0}$ as its initial data. From the dominated convergence Theorem, $u_{n}(\cdot, 0) \rightarrow \tilde{u}_{0}$ in $L_{l o c}^{1}(\mathbb{R})$, hence from $L^{1}$ contractivity, $u_{n}(x, T) \rightarrow$ $\tilde{u}(x, T)$ a.e. $x \in \mathbb{R}$. Therefore for a.e. $x \in \mathbb{R}$,

$$
f^{\prime}(\tilde{u}(x, T))=\frac{x-\tilde{\rho}_{0}(x)}{T} .
$$

Hence the Lemma.

Proof of Theorem 3.2. From Lemma 3.5, there exists an initial data $\tilde{u}_{0}$ and the corresponding solution $\tilde{u}$ such that

$$
\begin{aligned}
f^{\prime}(\tilde{u}(x, T)) & =\frac{x-\tilde{\rho}_{0}(x)}{T} \text { if } x \in\left[\tilde{C}_{1}, \tilde{C}_{2}\right], \\
\tilde{u}_{0}(x) & =\theta_{f} \text { if } x \notin\left[\tilde{C}_{1}, \tilde{C}_{2}\right] .
\end{aligned}
$$

Since $f^{\prime}\left(a_{1}\right)=f^{\prime}\left(a_{2}\right)=0$. Therefore $u_{0} \in A$ and hence from (3.14)

$$
\inf _{w_{0} \in A} J\left(w_{0}\right) \leq J\left(\tilde{u}_{0}\right)=\bar{J}\left(\tilde{\rho}_{0}\right)=\inf _{\rho \in B} \bar{J}(\rho) \leq \inf _{w_{0} \in A} J\left(w_{0}\right) .
$$

Hence $\tilde{u}_{0}$ is an optimal solution to the problem and this proves the Theorem. 
4. Generalization. We extend the optimal control problem for the case when the target function can take arbitrary values out side a compact set and we generalized to initial and boundary value problem. Also same method works for $L^{p} \operatorname{cost}$ functional. Remark 4.1 In the optimal Control Problem, condition on $k$ can be relaxed and is as follows. We can assume that for $x \notin\left(C_{1}, C_{2}\right)$

$$
f^{\prime}(k(x))=\left\{\begin{array}{lll}
\alpha_{1} & \text { if } x<C_{1}, \\
\alpha_{2} & \text { if } x>C_{2} .
\end{array}\right.
$$

Then the class $B$ is defined as follows. Let $\rho \in L_{\text {loc }}^{\infty}(\mathbb{R})$ be such that

(i). $\rho$ is non decreasing function

(ii). There exist $A_{1}<A_{2}$ such that

$$
\rho(x)=\left\{\begin{array}{lll}
x-\alpha_{1} T & \text { if } & x<A_{1} \\
x-\alpha_{2} T & \text { if } & x>A_{2}
\end{array}\right.
$$

Let

$$
B=\{\rho ; \rho \text { satisfying (i) and (ii) }\} .
$$

Then by the similar arguments one can show that there exist a unique $\tilde{\rho} \in B$ such that

$$
J(\tilde{\rho})=\inf _{\rho \in B} J(\rho) .
$$

Then using the backward algorithm as earlier we get the corresponding solution for the optimal control problem which can be compute easily.

Remark 4.2 In view of the controllability of initial and boundary value problems [1], we can extend the optimal controllability for the boundary value problem. To illustrate this, let us consider one sided initial boundary value problem. Let $0<T, 0<C$, and $k \in L_{\text {loc }}^{2}(\mathbb{R})$ such that $k(x)=\theta_{f}$ for $x$ large. Let $u_{0} \in L^{\infty}, b \in L^{\infty}(0, T)$ and $u$ be the solution of (1.2) in $\Omega=(0, \infty) \times(0, T)$ with

$$
\begin{gathered}
u(t, 0)=b(t) \quad 0<t<T, \\
u(x, 0)=u_{0}(x) \quad x>0, \\
J\left(u_{0}, b\right)=\int_{\mathbb{R}} \mid f^{\prime}\left(u(x, T)-\left.f^{\prime}(k(x))\right|^{2} d x .\right.
\end{gathered}
$$

In order to make integral finite assume $u_{0}(x)=\theta_{f}$ for $x$ large. Hence define

$$
A=\left\{\left(u_{0}, b\right) \in L^{\infty}(\mathbb{R}) \times L^{\infty}(0, T) ; u_{0}(x)=\theta_{f}, \text { for large } x\right\} .
$$

Then optimal control problem is to find $\left(\tilde{u}_{0}, \tilde{b}\right)$ such that

$$
J\left(\tilde{u}_{0}, \tilde{b}\right)=\inf _{\left(u_{0}, b\right) \in A} J\left(u_{0}, b\right) .
$$

From Joseph-Gowda [9] and Lax - Olienik [7] formulas for any $\left(u_{0}, b\right) \in A$. there exist $0 \leq C_{1} \leq C$ and $\rho_{1}:\left[0, C_{1}\right] \rightarrow[0, T]$ a non increasing function and $\rho_{2}:\left[C_{1}, C\right] \rightarrow \mathbb{R}$ a non decreasing function such that 
(i). $\rho_{2}(x)=x$ for $x$ large and

$$
f^{\prime}(u(x, T))=\frac{x-\rho_{2}(x)}{T} \quad \text { if } \quad x \in\left(C_{1}, C\right) .
$$

(ii). $f^{\prime}(u(x, t))=\frac{x}{T-\rho_{1}(x)}, \quad 0<x<C_{1}$ and

$$
\left|\frac{x}{T-\rho(x)}\right| \leq \wedge
$$

where $\wedge$ is a constant depending on the Lipschitz constant of $f$ on $\left[-\|b\|_{\infty},\|b\|_{\infty}\right]$.

Therefore

$$
\begin{aligned}
J\left(u_{0}, b\right)= & \int_{0}^{C_{1}}\left|\frac{x}{T-\rho_{1}(x)}-f^{\prime}(k(x))\right|^{2} d x+\int_{C_{1}}^{C}\left|\frac{x-\rho_{2}(x)}{T}-f^{\prime}(k(x))\right|^{2} d x \\
= & J_{1}\left(\rho_{1}, C_{1}\right)+J_{2}\left(\rho_{2}, C_{1}\right) \\
= & J\left(\rho_{1}, \rho_{2}, C_{1}\right) \\
& m=\inf _{u_{0}, b} J\left(u_{0}, b\right)=\inf _{\rho_{1}, \rho_{2}, C_{1}}\left\{J_{1}\left(\rho_{1}, C_{1}\right)+J_{2}\left(\rho_{2}, C_{1}\right)\right\} .
\end{aligned}
$$

Let $\left(\rho_{1, k}, \rho_{2, k}, C_{1, k}\right)$ be a minimizing sequence. As in the previous case, it follows that there exists $A>0$, such that $\rho_{2, k}(x)=x$ for all $k$ and $x \geq A$. Hence $\left\{\rho_{i_{k}}\right\}$ are uniformly bounded monotone functions, therefore from Helly's theorem there exist a subsequence still denoted by $\left\{\rho_{1, k}, \rho_{2, k}, C_{k}\right\}$ converges $\left(\tilde{\rho}_{1}, \tilde{\rho}_{2}, \tilde{C}\right)$ for all $x \in[0, C]$. Hence by Fatau's Lemma $\left(\tilde{\rho}_{1}, \tilde{\rho}_{2}, \tilde{C}\right)$ is an optimal solution and from [1] we can construct the solution $\left(\tilde{u}_{0}, \tilde{b}\right)$.

If $\tilde{C}$ is known, then $\tilde{\rho_{1}}$ and $\tilde{\rho_{2}}$ can be obtained from the $L^{2}$ - projection as follows: Let $\eta_{1}(x)=\frac{x}{T-\rho_{1}(x)}$ then $\frac{x}{\eta_{1}} \leq T, \frac{\eta_{1}(x)}{x}$ is a non increasing right continuous function. Let $\eta:[0, C] \rightarrow \mathbb{R}$ be function such that

1. $\frac{\eta(x)}{x}$ is non increasing right, continuous function in $[0, \tilde{C}]$ and $\frac{x}{\eta(x)} \leq T$ in $[0, \tilde{C}]$.

Let

2. $\left.\eta\right|_{(\tilde{C}, C)}$ is non decreasing function.

$$
K_{N}=\{\eta ; \eta \text { satisfying (1), (2) and } 0 \leq \eta \leq N\} .
$$

Then $K_{N}$ is a closed convex set in $L^{2}((0, C))$ and let $\tilde{\eta}_{k} \in K_{N}$ such that

$$
J(\tilde{\eta})=\inf _{\eta \in K_{N}} J(\eta)=\inf \left\{\int_{0}^{\tilde{C}}\left|\eta(x)-f^{\prime}(k(x))\right|^{2} d x+\int_{\tilde{C}}^{C}\left|\frac{x-\eta(x)}{T}-f^{\prime}(k(x))\right|^{2} d x\right\} .
$$

Then for large $\eta$, if we define

$$
\begin{aligned}
& \tilde{\rho}_{1}(x)=T-\frac{x}{\tilde{\eta}(x)} \text { for } \quad x \in(0, \tilde{C}) \\
& \tilde{\rho}_{2},(x)=\tilde{\eta}(x) \quad \text { for } \quad x \in\left(\tilde{C}_{1}, C\right)
\end{aligned}
$$

then $\left(\tilde{\rho}_{1}, \tilde{\rho_{2}}, \tilde{C}\right)$ is the optimal solution. 
Remark 4.3 Let $1<p<\infty$ and $k$ be a measurable function satisfying (3.5) and $f^{\prime}(k) \in L^{p}(\mathbb{R})$. Let $u_{0} \in A$ and define the cost functional

$$
J_{p}\left(u_{0}\right)=\int_{\mathbb{R}}\left|f^{\prime}(u(x, T))-f^{\prime}(k(x))\right|^{p} d x .
$$

Then from Lax-Oleinik formula, there exists a $\rho \in B$ such that

$$
J_{p}\left(u_{0}\right)=\bar{J}_{p}(\rho)=\int_{\mathbb{R}}\left|\frac{x-\rho(x)}{T}-f^{\prime}(k(x))\right|^{p} d x
$$

and

$$
\inf _{u_{0} \in A} J_{p}\left(u_{0}\right)=\inf _{\rho \in B} \bar{J}_{p}(\rho)
$$

Then as in Lemma 3.3

$$
\inf _{\rho \in B} \bar{J}_{p}(\rho)=\inf _{\rho \in \tilde{B}_{0}} \bar{J}_{p}(\rho) .
$$

and $\tilde{B}_{0} \subset L^{p}\left(\left[\tilde{C}_{1}, \tilde{C}_{2}\right]\right)$ is a closed convex set. Hence from uniform convexity of $L^{p}$ spaces. there exists a unique $\tilde{\rho}_{0} \in \tilde{B}_{0}$ such that

$$
\bar{J}_{p}\left(\tilde{\rho}_{0}\right)=\inf _{u_{0} \in \tilde{B}_{0}} \bar{J}_{p}(\rho) .
$$

\section{REFERENCES}

[1] Adimurthi, Shyam Sundar Ghoshal, G.D.Veerappa Gowda, Exact controllability of scalar conservation law with strict convex flux - preprint , 2011.

[2] Adimurthi, Shyam Sundar Ghoshal, G.D.Veerappa Gowda, Structure of the entropy solution of a conservation law with strict convex flux, - preprint, 2011.

[3] C. Bardos and O. Pironneau, Derivatives and control in presence of shocks, Computational Fluid Dynamics Journal 11(4) (2003) 383-392.

[4] A. Bressan and A. Marson, A maximum principle for optimally controlled systems of conservation laws, Rend. Sem. Mat. Univ. Padova 94 (1995) 79-94.

[5] C.Castro, F.Palacios, E.Zuazua, Optimal control and vanishing viscosity for the Burgers equations, Integral methods in science and engineering, vol 2, 65-90, Birkhouser Boston Inc, Boston MA 2010.

[6] C.Castro, E.Zuazua, Flux identification for 1-d scalar conservation laws in the presence of shocks, Math.Comp. 80(2011),no.276,(2025-2070).

[7] L.C. Evans, Partial differential equations, Graduate studies in Mathematics, vol 19, AMS 1998.

[8] E.Godleweski, P.A.Raviant, Hyperbolic systems of conservation laws, Mathematiques and Applications 3/4, ellipses, Paris, 1991.

[9] Joseph.K.T, Veerappa Gowda, G.D, Explicit formula for the solution of Convex conservation laws with boundary condition, Duke Math.J..62 , No.2, 1991, 401-416.

[10] P.Lax , Hyperbolic systems of conservation Laws II, comm Pure Appl. Math, 10 ( 1957), 535566. 